12

\title{
Моделирование неоднородных электронных потоков в электронно-оптической системе гиротрона
}

\author{
(C) О.И. Лукша, П.А. Трофримов \\ Санкт-Петербургский политехнический университет Петра Великого, \\ 195251 Санкт-Петербург, Россия \\ e-mail: louksha@rphf.spbstu.ru
}

(Поступило в Редакцию 14 сентября 2017 г.)

Получены новые расчетные данные о влиянии эмиссионных неоднородностей на качество электронного пучка, формируемого в электронно-оптической системе гиротрона. В расчетах использовались распределения плотности тока эмиссии, измеренные для разных катодов в гиротроне СПбПУ. Показано удовлетворительное соответствие экспериментальных и расчетных данных по влиянию эмиссионных неоднородностей на скоростной разброс электронов. Продемонстрирована необходимость учета реального распределения плотности тока эмиссии по поверхности катода для определения основных параметров электронного потока скоростного и энергетического разброса электронов, пространственной структуры пучка, коэффициента отражения электронов от магнитной пробки. Обсужден предельный уровень эмиссионных неоднородностей, допустимый для эффективной работы гиротронов.

DOI: 10.21883/JTF.2018.04.45733.2484

\section{Введение}

Сегодня гиротроны являются наиболее эффективными мощными генераторами когерентного излучения в миллиметровом и субмиллиметровом диапазонах длин волн. Они применяются для нагрева плазмы и управления током в установках управляемого термоядерного синтеза, при обработке материалов, в спектроскопии высокого разрешения и в других приложениях. Эффективность и предельные достижимые параметры гиротронов, как и других вакуумных СВЧ приборов, определяются качеством электронного пучка, поступающего в область его взаимодействия с высокочастотным полем. Применительно к гироприборам требуются винтовые электронные потоки (ВЭП), в которых сочетаются высокое значение осцилляторной энергии электронов с малым скоростным и энергетическим разбросом и поперечной структурой, обеспечивающей эффективное взаимодействие пучка с рабочей модой (например, $[1,2]$ ).

Важным фактором, влияющим на качество электронного пучка в гироприборах, является неоднородность термоэлектронной эмиссии с катода магнетронноинжекторной пушки (МИП), которая обычно используется в качестве источника электронов в таких приборах. Как правило, эти пушки работают в режиме температурного ограничения эмиссии. Поэтому неоднородности работы выхода и температуры приводят к неоднородному распределению плотности тока в поперечном сечении ВЭП. Это является причиной появления неоднородных полей, которые, в свою очередь, увеличивают скоростной разброс электронов, способствуют возбуждению паразитных колебаний пространственного заряда и появлению разброса электронов по энергии (например, [3-12]). Неоднородная структура пучка ответственна также за неоднородный нагрев коллектора и развитие паразитных мод в резонаторе [13]. Особенностями термокатодов, применяемых в мощных гироприборах, является сложная форма и достаточно большая площадь эмитирующей поверхности, что обусловливает технологические трудности их изготовления. Кроме того, в процессе эксплуатации гиротрона возможно изменение эмиссионных характеристик катода, например, в результате ионной и электронной бомбардировок его поверхности [12].

Распространен метод оценки степени неоднородности термоэлектронной эмиссии, основанный на анализе переходного участка вольт-амперной характеристики (BAX) пушки между областями ограничения тока пространственным зарядом и температурного ограничения (например, [14]). Протяженность переходного участка BAX характеризует степень эмиссионной неоднородности, усредненную по всей поверхности катода. Простота и оперативность данного метода позволяют использовать его для контроля качества термокатода непосредственно в процессе эксплуатации лампы. В ряде лабораторий с помощью этого метода были проведены исследования катодов в различных устройствах гиротронного типа, что позволило получить данные о влиянии степени эмиссионной неоднородности катода на характеристики ВЭП и КПД гиротрона [3-6,10].

Однако наряду с величиной интегрального коэффициента эмиссионной неоднородности несомненный интерес представляет и информация о пространственном распределении эмиссионного тока по поверхности катода. Такая информация может быть использована для определения причин неоднородности эмиссии с катода, а также для уточнения влияния этой неоднородности на параметры ВЭП и на процессы взаимодействия пучка с электромагнитным полем в резонаторе. Программа по изучению „катодной проблемы“ в гиротронах, основанная на прямых измерениях распределений эмиссион- 
ного тока по поверхности катодов, была реализована в СПбПУ [12,15-21]. В нескольких приборах гиротронного типа, включая экспериментальный гиротрон СПбПУ с рабочей частотой $74.2 \mathrm{GHz}$ и выходной мощностью $100 \mathrm{~kW}$, были исследованы более 20 термокатодов, изготовленных по различным технологиям. В экспериментах определялись азимутальные распределения плотности тока эмиссии путем измерения тока, прошедшего через малое отверстие в аноде в отсутствии магнитного поля в зависимости от азимутального положения катодного блока прибора. Для исследования гиротронных катодов и регулирования их эмиссионных характеристик была также использована специальная вакуумная установка [22,23], в которой был отработан метод повышения эмиссионной однородности катодов, основанный на обработке участков с аномальной эмиссией с помощью ионной бомбардировки. Отметим, что подобная установка для диагностики гиротронных катодов была позже создана в фирме Calabazas Creek Research, Inc [11].

В экспериментах, проведенных в СПбПУ, была определена связь эмиссионной неоднородности катода с характеристиками паразитных низкочастотных колебаний (НЧК), обусловленными отражением части ВЭП от магнитной пробки и накоплением пространственного заряда в ловушке между катодом и резонатором гиротрона. Отметим, что в электронно-оптической системе (ЭОС) гиротрона транспортировка ВЭП между пушкой и резонатором осуществляется в нарастающем магнитном поле, где происходит накачка поперечной энергии электронов. В присутствии скоростного разброса электронов неизбежно отражение части ВЭП от магнитной пробки, что может сопровождаться возбуждением указанных выше паразитных колебаний и в целом негативно сказываться на качестве формируемого пучка. Полученные в СПбПУ экспериментальные данные позволили оценить величину скоростного разброса электронов в ВЭП, формируемых катодами с разной эмиссионной неоднородностью $[18,20]$. На основании этих данных был сделан вывод, что при коэффициенте неоднородности эмиссии $\delta j_{e}{ }^{1}$, превышающем 20-25\%, возрастание этих неоднородностей приводит к увеличению скоростного разброса электронов и снижению порога возбуждения низкочастотных колебаний пространственного заряда, а в присутствии этих колебаний - к увеличению их амплитуды и разброса электронов по энергии.

Новые сведения о закономерностях влияния эмиссионных неоднородностей катода на качество ВЭП в гиротроне могут быть получены в результате трехмерного траекторного анализа с использованием реальных распределений плотности эмиссии по поверхности катода, полученных в эксперименте. В настоящей работе подобный анализ был выполнен с использованием программного пакета 3D-моделирования CST

\footnotetext{
${ }^{1}$ Коэффициент $\delta j_{e}$ определялся по измеренной зависимости плотности тока эмиссии от азимутальной координаты $j_{e}(\theta)$ как относительное среднеквадратичное отклонение величины $j_{e}$ от среднего значения (например, [18]).
}

Таблица 1. Основные параметры гиротрона и характеристики его рабочего режима

\begin{tabular}{l|c}
\hline \multicolumn{1}{c|}{ Параметр } & Значение \\
\hline Ускоряющее напряжение & $U_{0}=30 \mathrm{kV}$ \\
Ток пучка & $I_{b}=10 \mathrm{~A}$ \\
Длительность импульса & $\tau=30-60 \mu \mathrm{s}$ \\
Индукция магнитного поля & $B_{0}=2.75 \mathrm{~T}$ \\
в области резонатора & \\
Индукция магнитного поля & $B_{c}=0.152 \mathrm{~T}$ \\
у катода & $\mathrm{TE}_{12,3}$ \\
Рабочая мода & $f_{0}=74.2 \mathrm{GHz}$ \\
Рабочая частота & $R_{0}=14.45 \mathrm{~mm}$ \\
Радиус резонатора & $R_{c}=35 \mathrm{~mm}$ \\
Средний радиус эмитирующего & \\
пояска катода &
\end{tabular}

Studio Suite [24]. Были рассчитаны параметры ВЭП, поступающего в резонатор гиротрона СПбПУ, для трех катодов с различными распределениями $j_{e}(\theta)$, которые ранее были использованы в экспериментах. Траекторный анализ был выполнен для двух типов магнетронноинжекторных пушек - со стандартной геометрией и со специальным управляющим электродом [25].

\section{Описание расчетной модели}

Основные параметры гиротрона СПбПУ приведены в табл. 1. Для формирования электронного пучка в этом приборе использовалась трехэлектродная МИП (рис. 1). При этом большинство экспериментов, а также описанные ниже расчеты были выполнены для „двухэлектродного“ режима работы пушки с заземленным анодом. В стандартной конфигурации пушки (рис. 1,a) угол наклона образующей конусной части катода к оси был одинаковым вдоль этой образующей и составлял $35^{\circ}$. В модифицированной версии пушки (рис. $\left.1, b\right)$ был установлен управляющий электрод с углом наклона конусной части, равным 50, и изолированный от других элементов катодного блока. Как показали эксперименты и 2D-моделирование [25], регулированием потенциала управляющего электрода можно добиться оптимизации распределения электрического поля в прикатодной области МИП и, как следствие, снижения скоростного разброса в пучке, повышения рабочего питч-фактора ${ }^{2}$ и увеличения КПД гиротрона.

В настоящей работе расчеты были выполнены для азимутальных распределений плотности тока эмиссии $j_{e}(\theta)$ (рис. 2), которые ранее были получены в экспериментах

\footnotetext{
${ }^{2}$ Величиной питч-фактора $\alpha=v_{\perp} / v_{\|}\left(v_{\perp}\right.$ и $v_{\|}-$поперечная и продольная компоненты скорости электрона) принято характеризовать долю сосредоточенной в поперечном движении электронов энергии, из которой „черпается“ энергия выходного СВЧ излучения в гирорезонансных приборах. Как правило, значения среднего по ансамблю электронов питч-фактора в рабочих режимах мощных гиротронов не превышают 1.2-1.4 (например, [1]).
} 


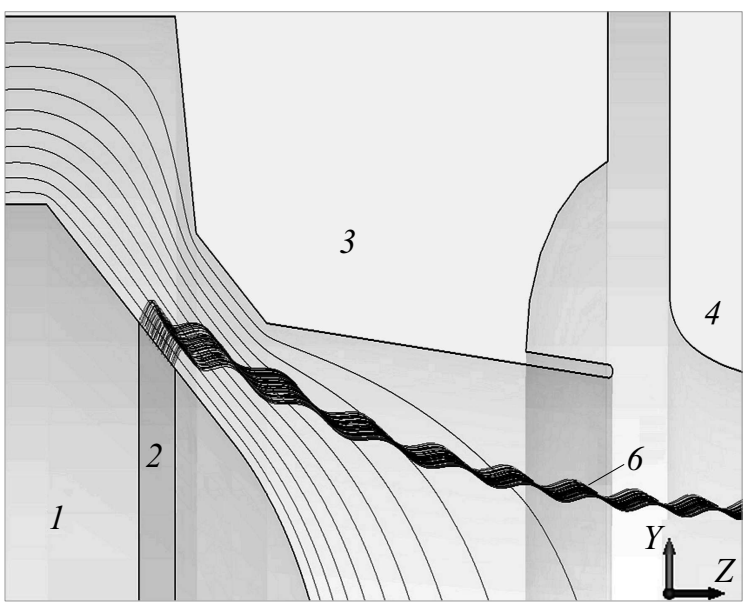

$b$

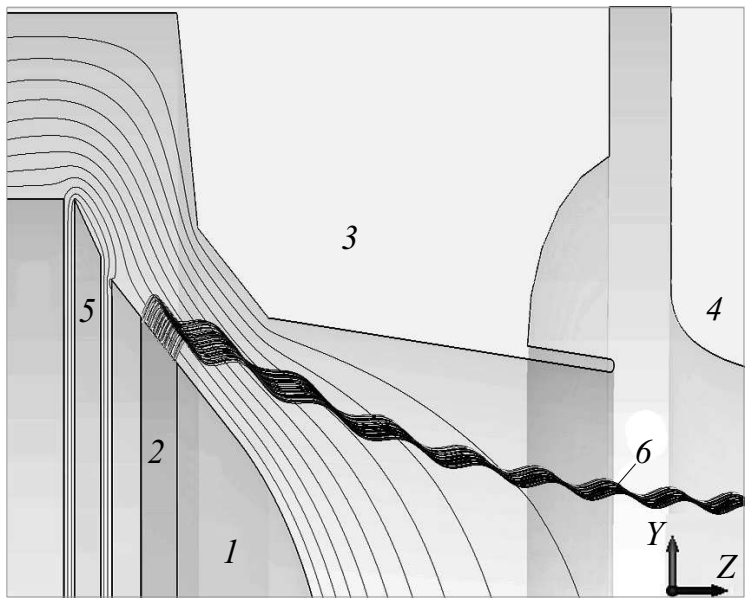

Рис. 1. Модель МИП гиротрона для стандартной геометрии катода $(a)$ и для катода с управляющим электродом $(b) .1-$ катод, 2 - эмитирующий поясок, 3 - анод, 4 - канал дрейфа пучка, 5 - управляющий электрод. Показаны эквипотенциальные линии в области между катодом и анодом и траектории электронов 6, рассчитанные для рабочего режима гиротрона (для схемы с управляющим электродом $(b)$ напряжение $\left.U_{\text {cont }}=-9 \mathrm{kV}\right)$.

с $\mathrm{LaB}_{6}$-катодами $\mathrm{C} 1, \mathrm{C} 2$ и металлопористым W-Baкатодом С3 (см., например, [18]). Высокотемпературные $\mathrm{LaB}_{6}$-катоды демонстрировали относительную устойчивость при работе в гиротроне. Для них было обнаружено, что в течение нескольких недель эксплуатации, когда лампа не подвергается развакуумированию и отсутствует заметное повышение давления вследствие пробоев, крупномасштабные особенности распределений $j_{e}(\theta)$, а также коэффициент эмиссионной неоднородности $\delta j_{e}$ изменяются незначительно. W-Ва-катоды были более чувствительны к воздействию вредных факторов. Из-за неоднородности нагрева термопояска, а также вследствие неоднородностей потоков нейтральных и заряженных частиц, попадающих на его поверхность, начальные неоднородности плотности тока эмиссии нарастали с течением времени [12]. Распределение $j_{e}(\theta)$ для $\mathrm{W}-\mathrm{Ba}-$ катода С3, полученное на этапе его эксплуатации в гиротроне, показано на рис. 2, c. На этом рисунке приведено также более плавное моделирующее распределение $j_{e}(\theta) \propto \sin (\theta)+0.3 \sin (3 \theta)$ с аналогичным коэффициентом эмиссионной неоднородности $\delta j_{e}=74 \%$ (катод Mod).

Самосогласованные электронные траектории в ЭОС гиротрона рассчитывались с помощью вычислителя Tracking Solver, входящего в программный модуль CST Particle Studio (CST PS). Параметры ВЭП (среднее значение питч-фактора, разброс скоростей электронов и др.), обсуждаемые ниже, регистрировались с помощью специального монитора, установленного на расстоянии $z=260.5 \mathrm{~mm}$ от центра эмитирующего пояска катода в области максимума распределения $B(z)$, где располагается резонатор гиротрона. Для моделирования неоднородностей эмиссии эмитирующий поясок катода разбивался на 72 сектора, смещенных друг относительно друга в азимутальном направлении. Ток с каждого из секторов в модели с фиксированной эмиссией задавался в соответствии с экспериментальной зависимостью $j_{e}(\theta)$, измеренной с шагом $\Delta \theta=5^{\circ}$ (рис. 2). Каждый из секторов был источником 50 крупных частиц с примерно одинаковым током, что в сумме давало 3600 электронных траекторий.

Для верификации расчетных данных было произведено сравнение результатов траекторного анализа, полученных с помощью двух кодов CST PS и EGUN [26] для одной и той же модели при одинаковых размерах ячеек сетки $l=0.4 \mathrm{~mm}$. В результате 2D-моделирования с помощью программы EGUN в гиротроне со стандартной МИП были получены следующие значения основных параметров ВЭП: средний питч-фактор $\bar{\alpha}=1.289$, разброс поперечной скорости $\delta v_{\perp}=6.50 \%$. Отметим, что величины скоростного и энергетического разбросов в рамках данного исследования определялись как среднеквадратичные отклонения от их средних значений. Те же параметры, полученные в результате моделирования в CST PS для катода с однородным распределением эмиссии (одинаковый ток со всех секторов), равнялись соответственно 1.277 и 6.93\%. Данные, полученные с помощью двух указанных программ при одинаковом сеточном разбиении, удовлетворительно согласуются друг с другом. Отметим, что при выбранном размере ячеек сетки $l=0.4 \mathrm{~mm}$ область моделирования в программе CST PS разбивалась примерно на 80 млн. ячеек.

\section{Результаты моделирования}

Данные о параметрах ВЭП, рассчитанных для катодов $\mathrm{C} 1, \mathrm{C} 2, \mathrm{C} 3$, а также для катодов Uniform с однородной эмиссией и Mod с моделирующим распределением $j_{e}(\theta)$, 
Таблица 2. Параметры ВЭП для катодов с разной эмиссионной неоднородностью

\begin{tabular}{c|c|c|c|c|c}
\hline \multicolumn{7}{c}{ МИП со стандартной геометрией } \\
\hline Катод & $\delta j_{e}, \%$ & $\bar{\alpha}$ & $\delta v_{\perp}, \%$ & $\delta \varepsilon, \%$ & $K_{\text {ref, } \%}$ \\
\hline Uniform & 0 & 1.277 & 6.93 & 0.26 & - \\
C1 & 23 & 1.279 & 7.04 & 0.50 & - \\
C2 & 50 & 1.283 & 7.52 & 1.33 & - \\
C3 & 74 & 1.288 & 8.07 & 2.10 & 0.26 \\
Mod & 74 & 1.283 & 8.09 & 2.63 & 0.1 \\
\hline
\end{tabular}

МИП с управляющим электродом $\left(U_{\text {cont }}=-9 \mathrm{kV}\right)$

\begin{tabular}{c|c|c|c|c|c}
\hline Катод & $\delta j_{e}, \%$ & $\bar{\alpha}$ & $\delta v_{\perp}, \%$ & $\delta \varepsilon, \%$ & $K_{\text {ref, } \%}$ \\
\hline Uniform & 0 & 1.288 & 3.05 & 0.28 & - \\
C1 & 23 & 1.288 & 3.29 & 0.50 & - \\
C2 & 50 & 1.290 & 4.26 & 1.34 & - \\
$\mathrm{C} 3$ & 74 & 1.294 & 5.35 & 2.10 & -
\end{tabular}

сведены в табл. 2. Здесь приведены значения коэффициента эмиссионной неоднородности $\delta j_{e}$, среднего питчфактора $\bar{\alpha}$, скоростного $\delta v_{\perp}$ и энергетического $\delta \varepsilon$ разбросов, а также коэффициента $K_{\text {ref, }}$ определяющего долю электронного заряда, отраженного от магнитной пробки. Моделирование ЭОС гиротрона с МИП стандартной геометрии было выполнено для рабочего режима, параметры которого указаны в табл. 1. Соответствующие траектории электронов, эмитированных одним из катодных секторов (катод Uniform), приведены на рис. 1, $a$. Из данных табл. 2 (верхняя часть) видно, что с ростом эмиссионных неоднородностей происходит увеличение скоростного и энергетического разбросов электронов в ВЭП. Очевидно, что в реальных условиях значения величин $\delta v_{\perp}$ и $\delta \varepsilon$ зависят от относительного вклада различных факторов разброса скоростей и энергий электронов. В рамках проведенного моделирования учитывались лишь факторы, связанные с действием статических полей, включая собственное поле пространственного заряда. Однако к дополнительному скоростному разбросу может также приводить шероховатость эмитирующей поверхности катода, неидеальности изготовления и юстировки элементов лампы и др. (см., например, [2]). Высокочастотные поля, связанные с развитием различных неустойчивостей в ВЭП, кроме повышения разброса скоростей, могут также вызывать заметный рост энергетического разброса электронов [12].

Представляет интерес сравнить рассчитанные значения скоростного разброса с аналогичными значениями, определенными для разных катодов экспериментально в гиротроне СПбПУ. В эксперименте переход, например, от катода С1 к катоду С3 сопровождался снижением порогового питч-фактора, при котором возбуждались паразитные колебания, с 1.31 до 1.11 , что позволило оценить значения разброса поперечных скоростей $\delta v_{\perp}$ для этих катодов в отсутствии низкочастотных колебаний (НЧК) $-8.8 \%$ для $\mathrm{C} 1$ и $11.8 \%$ для С $[18]$.
Отметим, что эта оценка была выполнена в предположении моноэнергетичности пучка и гауссовой функции распределения электронов по скоростям. В расчетах значения $\delta v_{\perp}$ составляли $7.04 \%$ и $8.07 \%$ для катодов $\mathrm{C} 1$ и С3 соответственно. При этом следует учесть, что переход к более неоднородному катоду сопровождается увеличением энергетического разброса электронов и ухудшением пространственной структуры пучка (см. ниже), что может влиять на характеристики НЧК, в частности уменьшать пороговый питч-фактор их возникновения. Тогда можно предположить, что приведенные выше оценки разброса поперечных скоростей электронов, полученные на основании экспериментальных данных о пороге возникновения НЧК, несколько завышены по сравнению с реальными значениями этого разброса в ВЭП гиротрона.

Для моделирования МИП с управляющим электродом (рис. $1, b)$ был выбран режим, при котором достигаются минимальные значения скоростного разброса для однородного эмиттера. Для снижения $\delta v_{\perp}$ необходимо подать
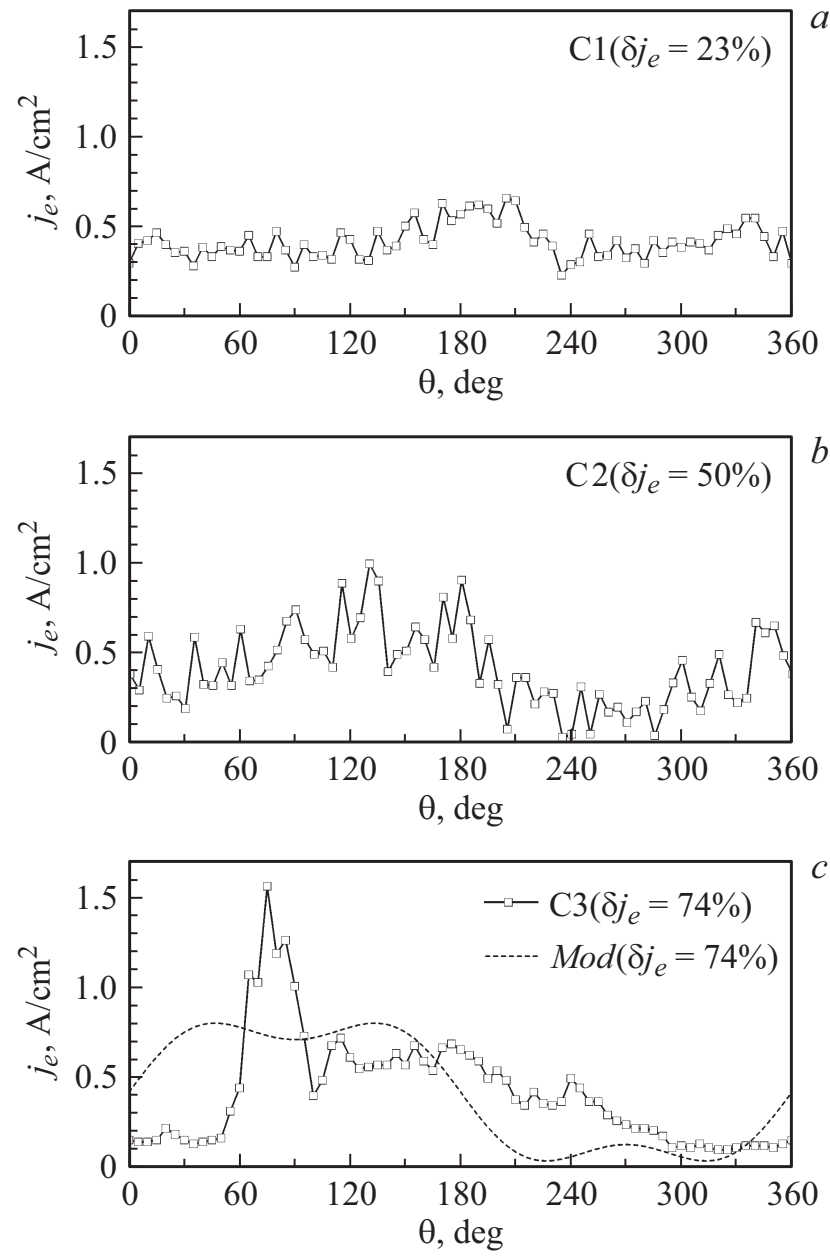

Рис. 2. Азимутальные распределения плотности тока эмиссии $j_{e}(\theta)$ и соответствующие коэффициенты эмиссионной неоднородности $\delta j_{e}$ для катодов $\mathrm{C} 1(a), \mathrm{C} 2(b)$ и $\mathrm{C} 3(c)$. Штриховой линией показано моделирующее распределение $j_{e}(\theta) \propto \sin (\theta)+0.3 \sin (3 \theta)$. 
отрицательное напряжение на управляющий электрод с тем, чтобы уменьшить позиционный разброс, проявляющийся в различии величин статических полей на траекториях электронов, вылетевших с разных точек эмиттера [25]. Однако подача отрицательного напряжения $U_{\text {cont }}$ приводит также к снижению среднего питчфактора электронов по сравнению со случаем $U_{\text {cont }}=0$. Для компенсации этого снижения был увеличен коэффициент магнитной компрессии за счет снижения магнитного поля на катоде. Описанные ниже результаты были получены при $U_{\text {cont }}=-9 \mathrm{kV}$ и $B_{c}=0.146 \mathrm{~T}$ (коэффициент перемагничивания $\left.B_{0} / B_{c}=18.78\right)$. Остальные параметры $\left(U_{0}, I_{b}, B_{0}\right)$, задающие режим работы гиротрона, не изменялись. Рассчитанные значения параметров ВЭП приведены в нижней части табл. 2, а электронные траектории - на рис. $1, b$.

Видно, что в МИП с управляющим электродом уменьшено значение начального позиционного разброса (катод Uniform) по сравнению со стандартной МИП. Как следствие, относительное влияние фактора скоростного разброса, связанного с эмиссионными неоднородностями, проявляется для этой пушки в большей степени (ср. верхнюю и нижнюю части табл. 2). Переход к МИП с управляющим электродом сопровождается также снижением порогового уровня эмиссионных неоднородностей, при котором они заметно влияют на суммарное значение разброса. Например, для стандартной МИП значение $\delta v_{\perp}$ для катода С1 $\left(\delta j_{e}=23 \%\right)$ лишь на $1.5 \%$ больше по сравнению с однородным катодом, что подтверждает оценки порогового коэффициента эмиссионной неоднородности для этой пушки, полученные на основании экспериментальных результатов $[18,20]$. Для МИП с управляющим электродом изменение величины $\delta v_{\perp}$ при переходе от однородного катода к катоду С1 более заметно и составляет $\sim 8 \%$.

Кроме увеличения разброса скоростей электронов, повышение эмиссионных неоднородностей приводит к изменению и других параметров ВЭП, определяющих его качество. В частности, увеличивается разброс электронов по энергиям $\delta \varepsilon$ (табл. 2). Для каждого из катодов значения $\delta \varepsilon$ примерно одинаковы для пушек с разной геометрией и определяются „провисанием“ потенциала ВЭП в области резонатора. Важно отметить, что даже небольшой разброс по энергии в несколько процентов приводит к заметному снижению КПД гиротрона (например, [27,28]). Следовательно, большой энергетический разброс может являться одной из основных причин пониженной эффективности работы гиротронов с сильно неоднородными катодами. Другая причина связана с появлением электронов, отраженных от магнитной пробки в области компрессии ВЭП. Отраженные частицы с суммарным зарядом, составляющим $0.26 \%$ от заряда всего пучка, были зарегистрированы при моделировании стандартной МИП с катодом С3 (табл. 2). При такой доле отраженных частиц накопление пространственного заряда в области между катодом и резонатором может приводить к развитию указанных выше паразитных НЧК, дополнительно снижающих качество ВЭП $[12,18,20]$.

Важным представляется исследованное влияние эмиссионной неоднородности на пространственную структуру ВЭП. Особенности азимутального распределения плотности электронного тока $j_{b}(\theta)$, обусловленные неоднородностью эмиссии, сохранялись в пучке при его транспортировке от катода до резонатора. Из-за азимутального дрейфа электронов в скрещенных радиальном электрическом и продольном магнитном полях происходило смещение этих особенностей по азимуту по мере увеличения продольной координаты $z$. В плоскости наблюдения $(z=260.5 \mathrm{~mm})$ распределение $j_{b}(\theta)$ было сдвинуто примерно на $25^{\circ}$ в сторону меньших значений азимутального угла $\theta$ по отношению к распределению $j_{e}(\theta)$ для исследованного рабочего режима гиротрона.

Положения центров ларморовских орбит частиц, прошедших в область резонатора, в плоскости поперечного сечения ВЭП показаны на рис. 3. Для каждого из приведенных распределений указано значение толщины стенки пучка $\Delta R_{g}$, как разницы между максимальным и минимальным значениями радиальных координат центров электронных орбит. Видно, что увеличение эмиссионной неоднородности сопровождается возрастанием величины $\Delta R_{g}$. При этом наблюдается смещение траекторий частиц как в сторону меньших, так и в сторону больших радиусов по отношению к исходным траекториям, рассчитанным для однородного катода. Из сравнения распределений, приведенных на рис. 2 и 3, можно сделать вывод, что радиальное смещение максимально в том диапазоне азимутальных координат $\theta$, в котором зависимость $j_{e}(\theta)$ имеет наибольший градиент. При таком сравнении следует учесть сдвиг ВЭП по азимуту на $\sim 25^{\circ}$ при движении электронов от катода до резонатора. Наиболее отчетливо радиальное смещение электронов проявляется для катода С3. Минимум распределения $R_{g}(\theta)$ для этого катода наблюдается при $\theta \approx 35$, а максимум - при $\theta \approx 65$ (рис. $3, d)$. При этом области с наибольшим градиентом $j_{e}(\theta)$ находятся с обеих сторон узкого участка аномально высокой эмиссии вблизи $\theta \approx 75$ (рис. 2,c). Причина уширения пучка связана с действием азимутального электрического поля, существующего в неоднородном ВЭП, которое в сочетании с продольным магнитным полем приводит к дрейфу электронов по радиусу. Амплитуда этого поля определяется градиентом зависимости $j_{e}(\theta)$ в выбранной области. Поэтому распределение электронов в плоскости поперечного ВЭП будет определяться не только перепадом плотности тока эмиссии с разных участков катода, но и пространственным масштабом неоднородностей эмиссии. Важно также отметить, что на пространственную структура полого ВЭП при неоднородной эмиссии с катода может дополнительно влиять развитие диокотронной неустойчивости, частным случаем которой является пространственная (статическая) диокотронная неустойчивость с частотой возмущений, равной нулю $[29,30]$. 

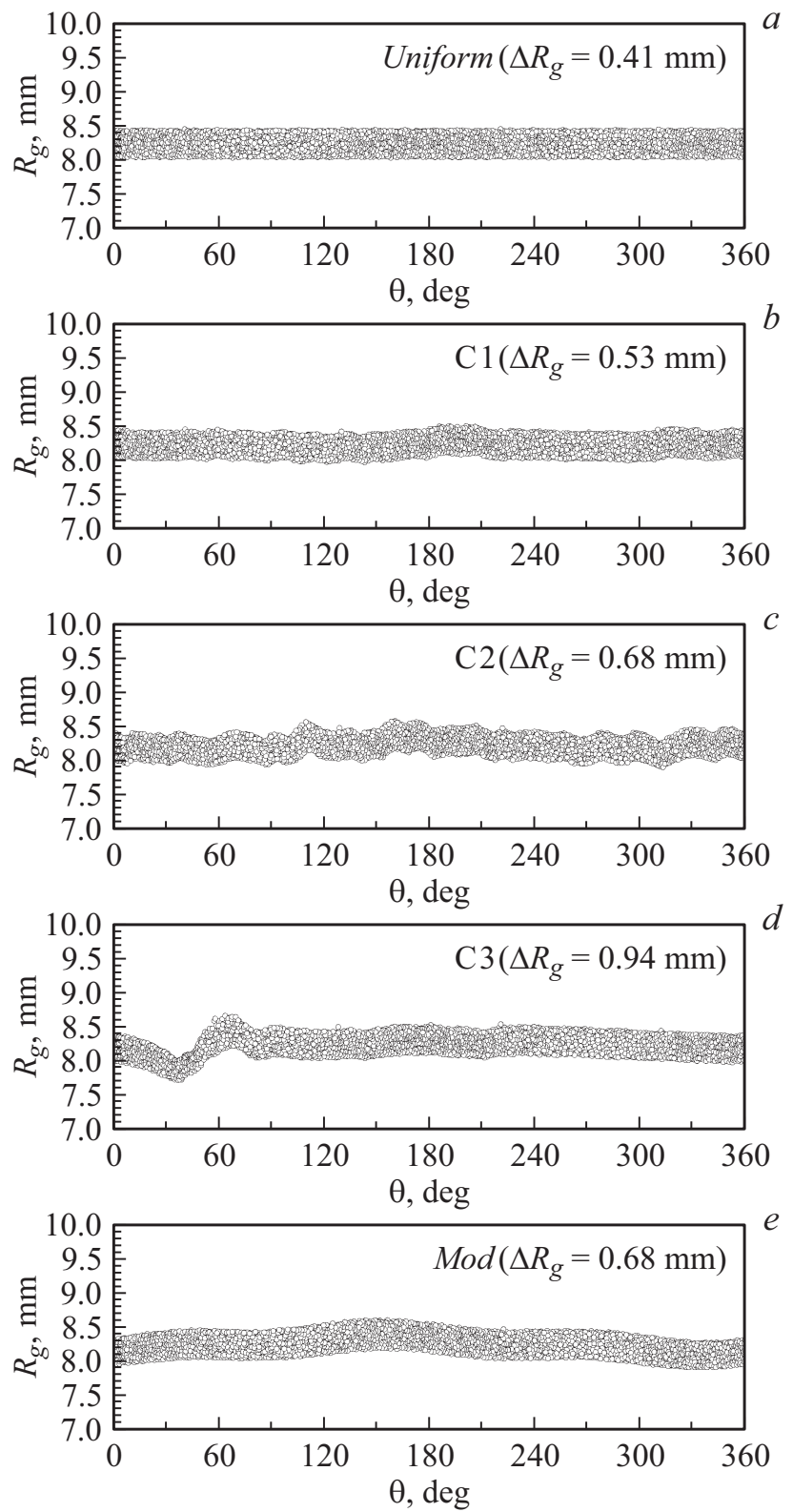

Рис. 3. Значения радиальных координат $R_{g}$ ведущих центров электронных траекторий в зависимости от азимута $\theta$ и значения толщины стенки ВЭП $\Delta R_{g}$ для катодов Uniform $(a), \mathrm{C} 1(b)$, $\mathrm{C} 2(c), \mathrm{C} 3(d), \operatorname{Mod}(e)$.

Результаты моделирования позволяют сделать вывод, что параметры ВЭП при неоднородной эмиссии с катода зависят от вида распределения $j_{e}(\theta)$. Для подтверждения этого вывода сравним данные для катодов С3 и Mod c одинаковым коэффициентом эмиссионной неоднородности $\delta j_{e}=74 \%$, полученные для стандартной геометрии МИП. Разброс скоростей электронов для этих катодов примерно одинаков (табл. 2). Он в основном определяется коэффициентом $\delta j_{e}$ и является следствием неоднородной экранировки пространственным зарядом внешнего электрического поля в области МИП [12]. При этом для обоих катодов имеется малое количество ча- стиц (три для С3 и две для Mod), которые испускаются с участков катода с аномально высокой эмиссией, приобретают в области МИП большую поперечную скорость и впоследствии отражаются от магнитной пробки.

Разброс энергий электронов $\delta \varepsilon$ больше для катода $\operatorname{Mod}$ по сравнению с С3. Величина $\delta \varepsilon$ с учетом вклада всех частиц определяется „провисанием“ потенциала пучка, который зависит от плотности тока нелинейно. Поэтому увеличение энергетического разброса электронов при переходе к более неоднородному катоду происходит не пропорционально возрастанию интегрального коэффициента эмиссионной неоднородности, как видно из табл. 2. Близкое к пропорциональному увеличение $\delta \varepsilon$ было отмечено только в случае, когда возрастала амплитуда неоднородностей и соответственно коэффициент $\delta j_{e}$, а вид зависимости $j_{e}(\theta)$ при этом сохранялся. Например, для распределения $j_{e}(\theta) \propto \sin (\theta)+0.3 \sin (3 \theta)$ величина $\delta \varepsilon$ равнялась $2.63 \%$ при коэффициенте неоднородности $\delta j_{e}=74 \%, 1.82 \%$ при $\delta j_{e}=50 \%$ и $0.87 \%$ при $\delta j_{e}=23 \%$.

Толщина стенки пучка $\Delta R_{g}$ максимальна для катода С3, что обусловлено наличием у него локальной области аномально высокой эмиссионной активности (рис. $2, c)$. Принято, что величина $\Delta R_{g}$, приемлемая для эффективной работы гиротрона, не должна превышать $\lambda / 6$, где $\lambda$ - длина волны излучения, в противном случае снижается эффективность генерации на рабочей моде и возможно возбуждение паразитных мод [31]. В нашем случае при $\lambda \approx 4 \mathrm{~mm}$ предельное значение $\Delta R_{g}$ равняется примерно $0.67 \mathrm{~mm}$. Для катодов С2 и $\mathrm{Mod}$ величина $\Delta R_{g}$ довольно близка к этому предельному значению, а для катода С3 - заметно его превышает.

\section{Заключение}

Таким образом, выполненное в настоящей работе моделирование показало важность учета реального распределения плотности тока эмиссии по поверхности катода при анализе влияния эмиссионных неоднородностей на качество электронного пучка, формируемого в электронно-оптической системе гиротрона. Новые расчетные данные о влиянии эмиссионных неоднородностей на скоростной разброс электронов, полученные с использованием распределений плотности эмиссионного тока для разных катодов в гиротроне СПбПУ, удовлетворительно согласуются с результатами экспериментов. Сравнение расчетных данных, полученных для гиротрона с пушками разной геометрии, позволяет сделать вывод, что предельный уровень эмиссионных неоднородностей, приемлемый для конкретного прибора, зависит от относительного вклада всех факторов, влияющих на основные параметры ВЭП - скоростной и энергетический разброс электронов, пространственную структуру пучка, коэффициент отражения от магнитной пробки. Показано, что для катодов с одинаковым интегральным коэффициентом эмиссионной неоднородности 
$\delta j_{e}$, но различным видом распределения $j_{e}(\theta)$ могут заметно различаться значения энергетического разброса электронов, толщины стенки пучка и коэффициента отражения от магнитной пробки.

Исследование выполнено за счет гранта Российского научного фонда (проект № 16-12-10010). Часть результатов была получена с использованием вычислительных ресурсов суперкомпьютерного центра СанктПетербургского политехнического университета Петра Великого (http://www.scc.spbstu.ru).

\section{Список литературы}

[1] Nusinovich G.S. Introduction to the physics of gyrotrons. Baltimore, MD: Johns Hopkins University Press, 2004. 335 p.

[2] Tsimring Sh.E. // Int. J. Infrared Millimeter Waves. 2001. Vol. 22. N 10. P. 1433-1468.

[3] Glyavin M.Yu., Kuftin A.N., Venediktov N.P. et al. // Int. J. Infrared Millimeter Waves. 1997. Vol. 18. N 11. P. $2137-$ 2146.

[4] Glyavin M.Yu., Goldenberg A.L., Kuftin A.N. et al. // IEEE Trans. Plasma Sci. 1999. Vol. 27. N 2. P. 474-483.

[5] Anderson J.P., Korbly S.E., Temkin R.J. et al. // IEEE Trans. Plasma Sci. 2002. Vol. 30. N 6. P. 2117-2123.

[6] Anderson J.P., Temkin R.J., Shapiro M.A. // IEEE Trans. Electron Devices. 2005. Vol. 52. N 5. P. 825-828.

[7] Advani R., Hogge J.P., Kreischer K.E. et al. // IEEE Trans. Plasma Sci. 2001. Vol. 29. N 6. P. 943-950.

[8] Kuftin A.N., Lygin V.K., Manuilov V.N. et al. // Int. J. Infrared Millimeter Waves. 1993. Vol. 14. N 4. P. 783-816.

[9] Pagonakis J.Gr., Vomvoridis J.L. // IEEE Trans. Plasma Sci. 2004. Vol. 32. N 3. P. 890-898.

[10] Zhang J., Illy S., Pagonakis I.G. et al. // IEEE Trans. Electron Devices. 2017. Vol. 64. N 3. P. 1307-1314.

[11] Ives R.L., Borchard P., Collins G. et al. // IEEE Trans. Plasma Sci. 2008. Vol. 36. N 3. P. 620-630.

[12] Лукша О.И. // Автореф. докт. дис., СПб., 2011. 285 с.

[13] Nusinovich G.S., Vlasov A.N., Botton M. et al. // Phys. Plasmas. 2001. Vol. 8. N 7. P. 3473-3479.

[14] Ходневич С.П. // Электронная техника. Сер. 1. Электроника СВЧ. 1969. Вып. 4. С. 118-130.

[15] Andronov A.N., Ilyin V.N., Luksha O.I. et al. // Dig. 20th Int. Conf. Infrared and Millimeter Waves. Orlando, USA, 1995. P. 141-142.

[16] Ilyin V.N., Louksha O.I., Mjasnikov V.E. et al. // Proc. 12th Int. Conf. High Power Particle Beams „BEAMS'98“. Haifa, Israel, 1998. Vol. 2. P. 800-804.

[17] Louksha O.I., Piosczyk B., Sominski G.G. et al. // Proc. 6th Int. Workshop Strong Microwaves in Plasmas. Nizhny Novgorod, Russia, 2005. Vol. 1. P. 135-140.

[18] Louksha O., Piosczyk B., Sominski G. et al. // IEEE Trans. Plasma Sci. 2006. Vol. 34. N 3. P. 502-511.

[19] Лукша О.И., Пиосчик Б., Соминский Г.Г. и др. // Изв. вузов. Радиофизика. 2006. Т. 49. Вып. 10. С. 880-886. (Luksha O.I., Piosczyk B., Sominski G.G. // Radiophys. Quantum Electron. 2006. Vol. 49. N 10. P. 793-798.)
[20] Лукша О.И., Самсонов Д.Б., Соминский Г.Г., Семин С.В. // ЖТФ. 2013. Т. 83. Вып. 5. С. 132-140. (Louksha O.I., Samsonov D.B., Sominskii G.G., Semin S.V. // Tech. Phys. 2013. Vol. 58. N 5. P. 751-759.)

[21] Louksha O.I., Sominski G.G., Arkhipov A.V. et al. // IEEE Trans. Plasma Sci. 2016. Vol. 44. N 8. P. 1310-1319.

[22] Louksha O., Piosczyk B., Sominski G., Thumm M. // Dig. Joint 31st Int. Conf. Infrared and Millimeter Waves and 14th Int. Conf. Terahertz Electronics. Shanghai, China, 2006. P. 86.

[23] Louksha O., Sominski G., Samsonov D. et al. // Dig. 35th IEEE Int. Conf. Plasma Science. Karlsruhe, Germany, 2008. P. 226.

[24] Электронный ресурс. Режим доступа: http://www.cst.com

[25] Лукша О.И., Самсонов Д.Б., Соминский Г.Г., Цапов А.А. // ЖТФ. 2012. Т. 82. Вып. 6. С. 101-105. (Louksha O.I., Samsonov D.B., Sominskii G.G., Tsapov A.A. // Tech. Phys. 2012. Vol. 57. N 6. P. 835-839.)

[26] Hermannsfeldt W.B. Electron trajectory program // SLAC Report 226, Stanford Linear Accelerator Center, Stanford University, 1979. 119 p.

[27] Dumbrajs O., Koponen J.P.T. // Phys. Plasmas. 1999. Vol. 6. N 6. P. 2618-2621.

[28] Завольский Н.А., Запевалов В.Е., Моисеев М.А. // Изв. вузов. Радиофизика. 2006. Т. 49. Вып. 2. С. 121-133. (Zavolsky N.A., Zapevalov V.E., Moiseev M.A. // Radiophys. Quantum Electron. 2006. Vol. 49. N 2. P. 108-119.)

[29] Лыгин В.К., Цимринг Ш.Е., Шевцов Б.И. // Изв. вузов. Радиофизика. 1991. Т. 34. Вып. 4. С. 419-425. (Lygin V.K., Tsimring Sh.E., Shevtsov B.I. // Radiophys. Quantum Electron. 1991. Vol. 34. N 4. P. 351-356.)

[30] Schuldt R., Borie E. // Int. J. Infrared Millimeter Waves. 1995. Vol. 16. N 10. P. 1675-1700.

[31] Pu R., Nusinovich G.S., Sinitsyn O.V., Antonsen T.M., jr. // Phys. Plasmas. 2010. Vol. 17. N 8. P. 083105-083105-6. 